\title{
Double Inverted Pendulum Based on LQG Optimal Control
}

\author{
ZHENG Yi ${ }^{1,2}$ \\ ${ }^{1}$ College of Mechanical and electrical engineering, Shandong \\ University of Science and Technology \\ Shandong, 266590, P.R. China \\ ${ }^{2}$ Qingdao Huang Hai University \\ Qingdao, Shandong, 266427 \\ fw.2004@163.com
}

\author{
ZHONG Peisi \\ Qingdao Huang Hai University \\ Qingdao, Shandong, 266427 \\ 1439669548@qq.com
}

\author{
YUE Qing Chao \\ Qingdao Huang Hai University \\ Qingdao, Shandong, 266427 \\ 121663260@qq.com
}

\begin{abstract}
In order to achieve the stable control for a double inverted pendulum system, linear quadratic Gauss optimal control was applied. Based on separation principle of LQG, firstly, the control law was proposed by the optimal control, and then a state feedback was obtained; whereas, the states of a system can't be measured completely, and the system could be inevitably affected by the a number of noise. For this reason, based on the rule of value of the corresponding parameters, the optimal estimations of the system outputs were received by means of Kalmar filtering. The conclusive experiment shown that the designed controller steadily dominated a double inverted pendulum system, and the system had a good ability of noise abatement and anti-interference.
\end{abstract}

Keywords-double inverted pendulum system; linear quadratic Gauss optimal control; state feedback; Kalmar filtering

\section{INTRODUCTION}

Inverted pendulum system is unstable system, it is typical multi-variable, strong coupling and nonlinear. So, in recent years, In order to verify some kind of control theory and method, the stability control of inverted pendulum and digital simulation become a typical scheme in the field of control. For example: The literature [1] proposed fuzzy control method that using hierarchical fuzzy neural network to control double inverted pendulum; Literature [2] used the linear quadratic optimal control theory, the closed-loop stability of the inverted pendulum has been realized; The literature [3] combined fuzzy control theory and optimal control theory, the fuzzy controller has been designed, the effective control of double inverted pendulum has been achieved. But, because of the LQR design based on state feedback, all the state variables in the system need to be known, it is very difficult in reality. Actual inverted pendulum system is also affected by the system noise and measurement noise, in order to solve these problems, Kalmar filter is used to reconstruct the system state. Using the double inverted pendulum as the research object, the mathematical model of the system is established, according to the principle of LQG separation[4], controller are designed. The design process, not only the effective for noise suppression is realized, in the control method, but also each parameter matrix and the parameters value rules is detailed description, to achieve the appropriate simulation results. Finally, the simulation results have been achieved.

\section{SySTEM MODELING}

For research object model is not too complicated, a simplified mathematical model is derived[5], the following hypothesis: the entire object in addition to the belt as a rigid body, and the swinging rod is a homogeneous rigid body; each part of the friction force is proportional to the relative velocity. Motor inductance is neglected. In Fig. $1 . r$ for the displacement of the car (right to positive direction); $\theta_{1}, \theta_{2}$ Angle between respectively swings, on stem and vertical (clockwise to positive direction); $r, r$ is speed and acceleration of the car; $\theta_{1}, \theta_{1}$ the angular velocity and angular acceleration of the draft link Respectively; $\dot{\theta}_{2}, \ddot{\theta}_{2}$ is angular velocity and angular acceleration of on the pole respectively; ${ }^{g}$ as the acceleration of gravity; $u$ as the input voltage power amplifier. According to the principle of the dynamics, the double inverted pendulum dynamics equation is get[6].

$$
\left[\begin{array}{lll}
M_{11} & M_{12} & M_{13} \\
M_{21} & M_{22} & M_{23} \\
M_{31} & M_{32} & M_{33}
\end{array}\right]\left[\begin{array}{c}
\ddot{r} \\
\ddot{\theta_{1}} \\
\ddot{\theta_{2}}
\end{array}\right]+\left[\begin{array}{ccc}
F_{11} & F_{12} & F_{13} \\
0 & F_{22} & F_{23} \\
0 & F_{32} & F_{33}
\end{array}\right]\left[\begin{array}{c}
\dot{r} \\
\dot{\theta_{1}} \\
\dot{\theta_{2}}
\end{array}\right]=\left[\begin{array}{c}
G_{1} \\
G_{2} \\
G_{3}
\end{array}\right](1)
$$

Among them: 
$M_{11}=M_{0}+M_{1}+M_{2}, M_{12}=\left(M_{1} l_{1}+M_{2} l_{1}\right) \cos \theta_{1}, M_{13}=M_{2} l_{2} \cos \theta_{2}$ Structure model of linear double inverted pendulum system as

$M_{21}=\left(M_{1} l_{1}+M_{2} l_{1}\right) \cos \theta_{1}, M_{22}=J_{1}+M_{1} l_{1}^{2}+M_{2} l_{2}^{2}, M_{23}=M_{2} L_{1} l_{2} \cos \left(\theta_{2}-\theta_{1}\right)$ shown in Fig. 1.

$M_{31}=M_{2} l_{2} \cos \theta_{2}, M_{32}=M_{2} l_{2} L_{1} \cos \left(\theta_{2}-\theta_{1}\right), M_{33}=J_{2}+M_{2} l_{2}^{2}$

$F_{11}=F_{0}, F_{12}=\left(M_{1} l_{1}+M_{2} L_{1}\right) \sin \theta_{1} \cdot \theta_{1}, F_{13}=M_{2} l_{2} \sin \theta_{2} \cdot \theta_{2}, F_{22}=F_{1}+F_{2}$

$F_{23}=M_{2} l_{2} L_{1} \sin \left(\theta_{2}-\theta_{1}\right) \theta_{2}+F_{2}, F_{32}=F_{2}-M_{2} l_{2} L_{1} \sin \left(\theta_{2}-\theta_{1}\right) \theta_{1}, F_{33}=F_{2}$

$G_{1}=\left(G_{0} u\right)^{T}, G_{2}=\left(\left(M_{1} l_{1}+M_{2} L_{1}\right) g \sin \theta_{1}\right)^{T}, G_{3}=\left(M_{2} l_{2} g \sin \theta_{2}\right)^{T}$

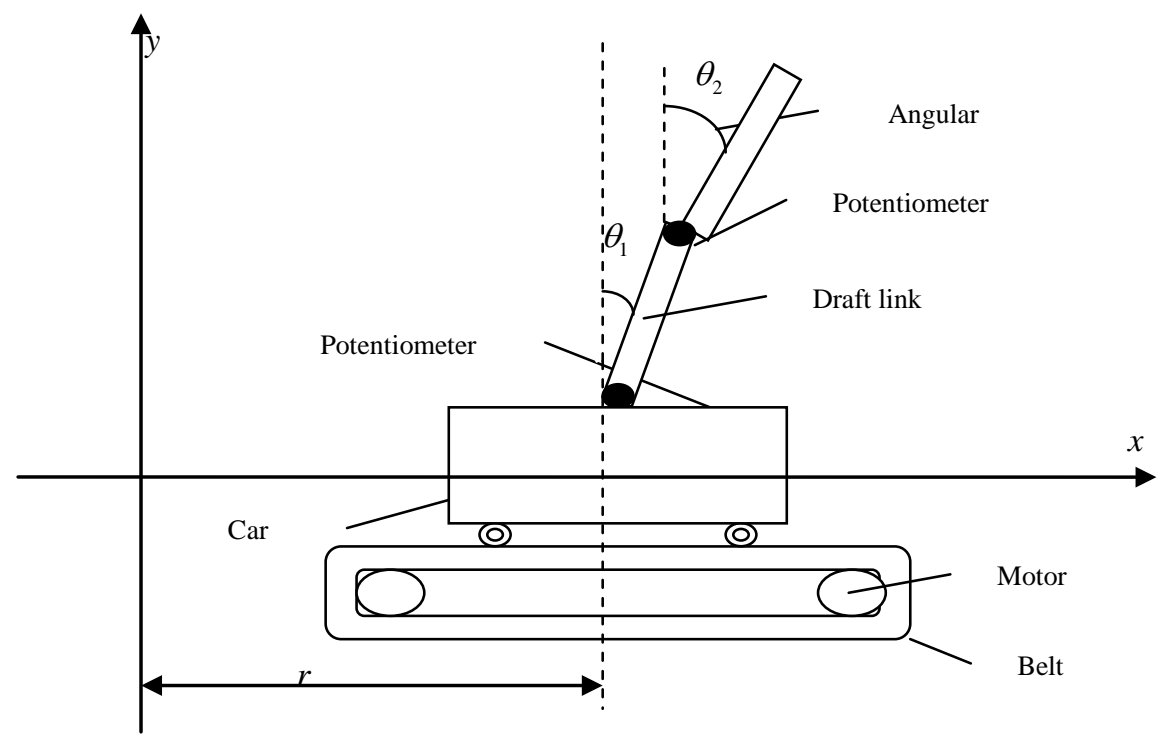

Fig. 1. Model structure of line double inverted pendulum

The meaning of the parameters and parameter values are shown in TABLE 1.

\begin{tabular}{|c|c|c|}
\hline parameter & Physical meaning/unit & The values \\
\hline$M_{0}$ & $\begin{array}{l}\text { The equivalent mass of the car system } \\
/ \mathrm{kg}\end{array}$ & 1.1023 \\
\hline$M_{1}$ & The quality of raft link/ kg & 0.1800 \\
\hline$M_{2}$ & the quality of on the pole / $\mathrm{kg}$ & 0.1250 \\
\hline$J_{1}$ & $\begin{array}{l}\text { moment of inertia on the center of mass } \\
\text { about raft link } /\left(\mathrm{kg}^{\cdot} \mathrm{m}^{\cdot} \mathrm{m}\right)\end{array}$ & 0.0036 \\
\hline$J_{2}$ & $\begin{array}{l}\text { moment of inertia on the center of mass } \\
\text { about on the pole } /(\mathrm{kg} \cdot \mathrm{m} \cdot \mathrm{m})\end{array}$ & 0.0034 \\
\hline$l_{1}$ & $\begin{array}{l}\text { the distance between the axis of rotation } \\
\text { and the center of mass about raft link } / \mathrm{m}\end{array}$ & 0.2850 \\
\hline$l_{2}$ & $\begin{array}{l}\text { the distance between the axis of rotation } \\
\text { and the center of mass about on the pole } / \mathrm{m}\end{array}$ & 0.2039 \\
\hline$L_{1}$ & $\begin{array}{l}\text { the distance between the axis of up and } \\
\text { down swings } / \mathrm{m}\end{array}$ & 0.4001 \\
\hline$F_{0}$ & $\begin{array}{l}\text { Friction coefficient of the car system } \\
/(\mathrm{N} \cdot \mathrm{s} / \mathrm{m})\end{array}$ & 20.1897 \\
\hline$F_{1}$ & $\begin{array}{l}\text { friction resistance coefficient of spindle } \\
\text { about on the pole } /(\mathrm{N} \cdot \mathrm{s} / \mathrm{m})\end{array}$ & 0.0069 \\
\hline$F_{2}$ & $\begin{array}{l}\text { friction resistance coefficient of spindle } \\
\text { about raft link } /(\mathrm{N} \cdot \mathrm{s} / \mathrm{m})\end{array}$ & 0.0021 \\
\hline$G_{0}$ & $\begin{array}{l}\text { The ratio of the force and the control } \\
\text { voltage } /(\mathrm{N} / \mathrm{V})\end{array}$ & 10.9812 \\
\hline
\end{tabular}


The equations are linearization around the system balance $\left(x=0 \quad\right.$ ), $\quad \sin \theta_{1} \approx \theta_{1}, \sin \theta_{2} \approx \theta_{2}$, $\cos \theta_{1} \approx 1, \cos \theta_{2} \approx 1$. On this basis, the definition of system state variables:

$$
x=\left[\begin{array}{llllll}
r & \theta_{1} & \left(\theta_{2}-\theta_{1}\right) & \dot{r} & \dot{\theta}_{1} & \left(\dot{\theta}_{2}-\dot{\theta}_{1}\right)
\end{array}\right]^{T}
$$

Then according to the type (1), making appropriate transformation system, state equation is obtained as follows:

$$
\left\{\begin{array}{l}
x=A x+B u \\
y=C x
\end{array}\right.
$$

Substitute into the parameters of the system in TABLE 1

$$
B=\left[\begin{array}{llllll}
0 & 0 & 0 & 6.2319 & -14.6941 & 16.8886
\end{array}\right]^{T}, C=\left[\begin{array}{llllll}
1 & 0 & 0 & 0 & 0 & 0 \\
0 & 1 & 0 & 0 & 0 & 0 \\
0 & 0 & 1 & 0 & 0 & 0
\end{array}\right]
$$

\section{CONTROL STRATEGIES}

\section{A. Control scheme described}

In order to achieve the steady pendulum control of double inverted pendulum system[7], using the optimal control of linear quadratic Gaussian (LQG) scheme, combining the LQR state feedback control and Kalmar state estimation, the control scheme is shown in Fig.2.

$$
A=\left[\begin{array}{cccccc}
0 & 0 & 0 & 1 & 0 & 0 \\
0 & 0 & 0 & 0 & 1 & 0 \\
0 & 0 & 0 & 0 & 0 & 1 \\
0 & -2.0001 & 0.0675 & -11.2781 & 0.0128 & 0.2568 \\
0 & 25.5321 & -12.7963 & 25.2639 & -0.2492 & 0.1667 \\
0 & -29.1352 & 56.2395 & -29.1427 & 0.5086 & -0.5023
\end{array}\right]
$$

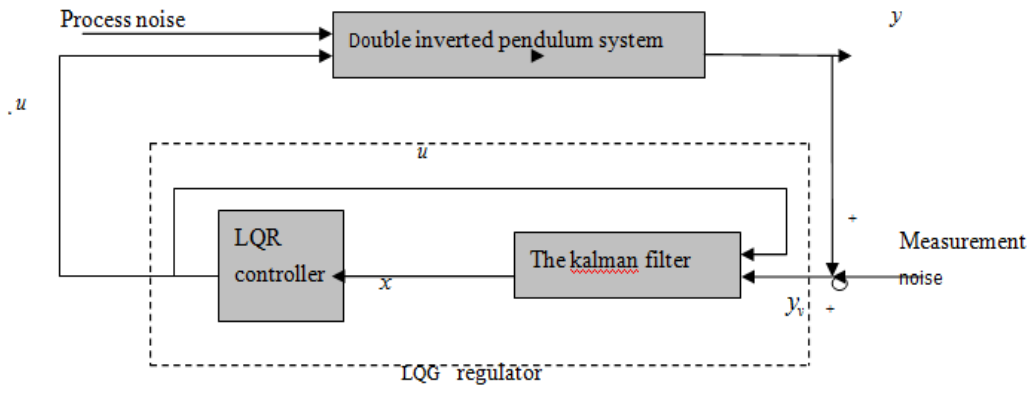

Fig. 2. The control scheme

\section{B. LQR controller}

According to the optimal control theory, firstly the controllability of the system need to determine, and control matrix is obtained by calculation:

$$
Q_{c}=\left[\begin{array}{llllll}
B & A B & A B^{2} & A B^{3} & A B^{4} & A B^{5}
\end{array}\right]
$$

It is a full rank. Then using the optimal control strategy to system (2), On the basis of state feedback $u=-K x$, looking for state feedback variables $K$, Make the performance index $\mathrm{J}$ to minimize[8]:

$$
J=\frac{1}{2} \int_{0}^{\infty}\left(x^{T} Q_{1} x+u^{T} R_{1} u\right) d t
$$

Among them: $Q_{1}, R_{1}$ for real symmetric positive definite and positive semi-definite matrix, and the system state variables and control the quantity of the weighted matrix; $K$ for the optimal feedback gain matrix, and meeting
$K=R_{1}^{-1} B^{T} P ; P$ is positive definite matrices and the following equation:

$$
P A+A^{T} P-P B R_{1}^{-1} B^{T} P+Q_{1}=0
$$

In the performance indicators,

$$
Q_{1}=\operatorname{diag}\left[\begin{array}{llllll}
Q_{11} & Q_{12} & Q_{13} & Q_{14} & Q_{15} & Q_{16}
\end{array}\right] \quad \text { The }
$$
specific guidelines can be explained as follows: In the simulation process, if the motion amplitude of the car and the pendulum rod is larger, increase the value of $Q_{14}, Q_{15}, Q_{16}$, reduce the value of the $Q_{11}, Q_{12}, Q_{13}$, Reduce the effect of proportional control corresponding, and increase the role of differential control. Through a lot of simulation experiments, found that within a certain range the greater the value of the matrix, the shorter adjustment of the system time. But when it's too much can cause larger dithering, so through continuous experiment, getting when 
$Q_{1}=\left[\begin{array}{llllll}700 & 600 & 700 & 200 & 150 & 100\end{array}\right], R_{1}=0.8$

the car and swinging rod can achieve good stability control, the optimal state feedback matrix:

$$
K=\left[\begin{array}{llllll}
29.6 & 769.9 & -1469.2 & 362.5 & 71.1 & 177.0
\end{array}\right]
$$

\section{The design of Kalmar filter}

In the reality, six state variables is not fully measured, in order to solve this contradiction, refactoring is needed. Disturbance of system state equation is as follows:

$$
\left\{\begin{array}{l}
x=A x+B u+T w \\
y_{v}=C x+v
\end{array}\right.
$$

Among them: $T$ for the noise input matrix; $y_{v}$ for the system of measuring output; $w$ and $v$ for the system noise and measurement noise, the mean to $0 ; Q$ and $R$ as covariance, and they are independent of each other respectively.

State estimation is made by the Kalmar filter:

$$
\left\{\begin{array}{l}
\hat{x}=(A-L C) \hat{x}+B u+L y \\
\hat{y}=C \hat{x}+v
\end{array}\right.
$$

Among them: $\hat{x}$ for State estimation of Kalmar filter; $\hat{y}$ for The output estimation of the filter ; $L=P C^{T} R^{-1}$, for the Kalmar filter gain. Under the condition of expectations

$$
P=\lim _{t \rightarrow \infty} E\left[(x-\hat{x})(x-\hat{x})^{T}\right] \text { for a minimum, the filter }
$$

for the optimal state estimation.

In the type (5), the noise input matrix, if its value is too large, the system is affected by noise, large variations in the value of system state variables, produce larger dithering; if the value is too small, and makes no noise disturbance. Therefore, according to the input and output amplitude range, taking the noise input matrix $T=\left[\begin{array}{llllll}0.14 & 0.05 & 0.05 & 0 & 0 & 0\end{array}\right]$, desirable system noise covariance $Q=1$, the measurement noise covariance $R=\left[\begin{array}{lllllllll}1 & 0 & 0 ; & 0 & 1 & 0 ; & 0 & 0 & 1\end{array}\right]$, you can get the Kalmar filter gain:

$$
L=\left[\begin{array}{ccc}
0.2054 & -0.2569 & 0.2107 \\
-0.2569 & 8.4519 & -2.4436 \\
0.2107 & -2.4436 & 14.1049 \\
0.0567 & -1.4516 & 1.6863 \\
-1.3014 & 38.7335 & -21.9498 \\
1.9434 & -33.2301 & 102.4796
\end{array}\right]
$$

Finally, according to the control scheme, using Kalmar estimator gain and the state feedback gain construct LQG controller scheme. The regulator equations as follows:

$$
\left\{\begin{array}{l}
u=-K \hat{x} \\
\hat{x}=(A-L C-B K) \hat{x}+L y_{v}
\end{array}\right.
$$

\section{THE SIMULATION VERIFICATION}

To use MATLAB simulation validation control effect of LQG controller scheme[9]. In the simulation: time interval value is too small, it can cause excessive state variables affected by noise, produce larger dithering; If the simulation time value is too small, the system may not achieve stability, the simulation results is not obvious. Therefore, through continuous trial and a lot of simulation experiments, $t_{s}=0.001 s, t=30 s$,input the noise in the system (5), getting the simulation curve of straight line double inverted pendulum system is obtained as shown in Fig. 2, 3.Can be seen from the final simulation results, the noise can be effectively suppressed, and the system after filtering has good robustness.
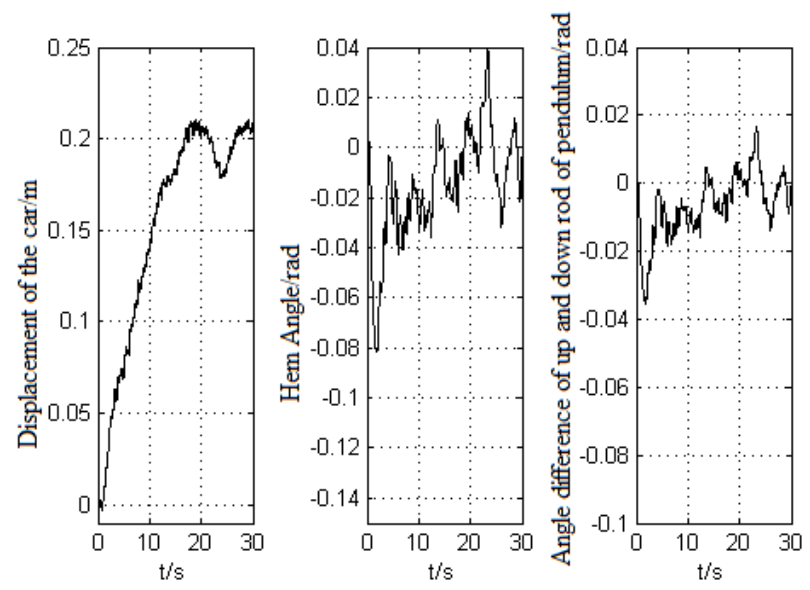

Fig. 3. The quantity of each state before LQG control scheme
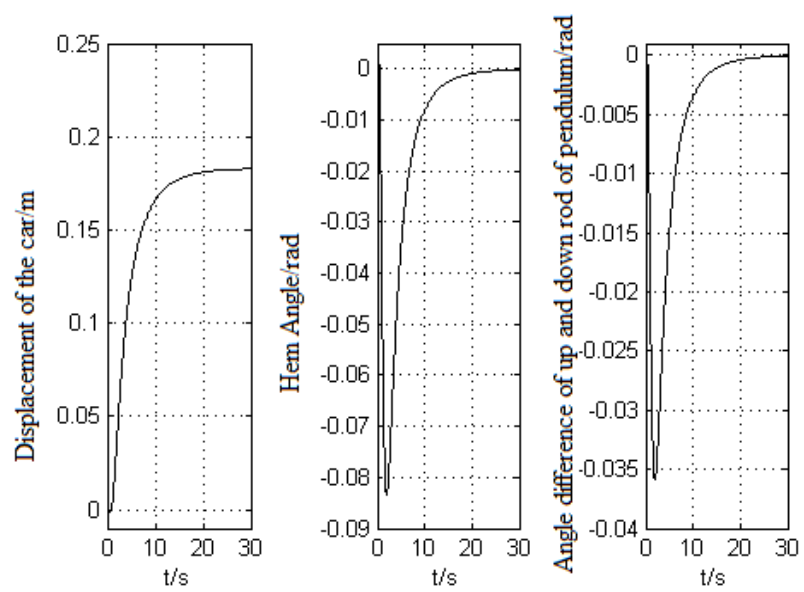

Fig. 4. The change of each state after the LQG control scheme

\section{CONCLUSION}

To achieve the stability of the double inverted pendulum control, on the basis of its mathematical model is set up, considering the system state is not fully measurable, and actual 
system will inevitably influenced by noise, so LQG method is adopted to research the scheme. According to the separation principle of the linear quadratic Gaussian optimal control, combining the state feedback gain and the Kalmar estimation gain which gets from the optimal control and the corresponding parameter value rule of the matrix are given in detail. The simulation indicates that control effect is better.

\section{ACKNOWLEDGMENT}

This paper aided financially by Shandong University of science and technology plan projects (J16LB58), Shandong province natural science foundation of China (2016ZRB01AIL), we thanks them greatly and honestly.

\section{REFERENCES}

[1] Zhen-Qing Yang, Su-Kang Cheng. Hierarchical fuzzy neural network control of double inverted pendulum [J]. Journal of motor and control, 2002, 6(3): 245-248.
[2] Chun-min Wang, Hong-Ying Yang. The design of the inverted pendulum control and simulation [J]. Journal of Jilin university (information science edition), 2009, 27(3): 242-247.

[3] Ling-Xia Jiao, He-Fang Zhang. Double inverted pendulum fuzzy control research [J]. Journal of electronic design engineering, 2009, 17(10): 105-107.

[4] Yan-Song Hou, Hua Li. A Kalmar filter based on the single inverted pendulum with LQR method [J]. Journal of Lanzhou Jiao tong university, 2005, 24(4):85-87,91.

[5] Guo-Xin LI, Yan-Li XU, Yong-zhou Jiang. The application of Kalmar filter in the inverted pendulum control system [J]. Journal of Changchun university, 2009,19(6):12-14.

[6] Pei-Pei Yu, Yong-Li Zhang, Hui-Feng Cheng. No trace of Kalmar filtering algorithm in the application research on the double inverted pendulum [J]. Fuzzy systems and mathematics, 2011, 25(5):158-163.

[7] Qian Qi, Zu-Shu Li, Yuan-Hong Dan, Lin Xiao. The inverted pendulum control system based on Kalmar filtering noise suppression [J]. Control and decision making, 2010, 25(8):1144-1148.

[8] Jian-Bin Ye, Hong-Wu Guo. Triple inverted pendulum LQG optimal control scheme of the application research [J]. Journal of computing technology and automation, 2011, 30(4): 9-13.

[9] Meng-yin Fu, Zhi-Hong Deng, Ji-Wei Zhang. Kalmar filtering theory and its application in navigation system [M]. Beijing: Science Press, 2003 